\title{
Investigating the Contents of the Senior Secondary School Chemistry Curriculum that can Inculcate Entrepreneurial Skills among Students in Nigeria
}

\author{
Ngozi Martina Eya \\ School of Science Education \\ Enugu State College of Education (Technical), Enugu \\ Nigeria
}

\begin{abstract}
Entrepreneurship or business creation and ownership has been revealed to be playing very important roles in the economic development of any country as it helps in alleviating the problem of unemployment of the citizens of the country. In this paper the contents of the senior secondary school curriculum which promotes the acquisition of entrepreneurial skills were identified. Also the different approaches that should be used in teaching chemistry to promote the inculcation of these skills were also identified. A total of fourty chemistry teachers drawn from thirty out of sixty-five government owned secondary schools and ten industrialists in Nsukka education zone constituted the sample. The instrument used was a self structured questionnaire. The results were presented on tables as well as the hypothesis tested with the t-test. The findings revealed that thirty three out of the forty eight major topics in the senior secondary school chemistry curriculum promote the acquisition of entrepreneurial skills while seventeen do not. Also the use of such approaches like field trip/excursion, laboratory work, inquiry approach, discovery approach and practical activities in teaching the contents will highly promote the inculcation of entrepreneurial skills among the students. However, there is no significant difference between scores from teachers and industrialists in the contents that can promote entrepreneurial skills by our students.
\end{abstract}

\section{Introduction}

The educational system of every country must have set goals relevant to the citizen's needs and national aspirations. Its major task lies in the process of how to ignite the latent talents in its citizens, prepare and thoroughly equip them with relevant knowledge and skills for viable functionality and ability to cope with any intellectual or cultural challenges which the rapidly changing environment may demand in future[3]. The perennial unemployment problem of school leavers in Nigeria is one of the variables that is rendering the education of our youths hopeless and thus dampens the interest of the youths in academic pursuit. This is because it lacks functionality and focus. This is why we have so many graduates that are roaming the streets of the nation seeking employment with certificates which most of them cannot defend. This unemployment has resulted in high incidence of various forms of social ills such as armed robbery, rape, housebreaking, political thuggery, car snatching, assassination, human trafficking diseases including Hiv/Aids [7]. No nation has ever survived successive high rates of unemployment because of the attendant waste of of human resources, which it breeds [6].Nigeria's future prosperity is a function of producing youths who are well prepared and well equipped to take their place in tomorrow's society. This involves implementing educational system that will equip youths and adults with saleable skills which can enable them become gainfully employed or self. employed . Such a system should provide for entrepreneurship education geared towards the production of potential entrepreneur.

Entrepreneurship according to Ikeme and Onu [9] is the effective manipulation of human intelligence as demonstrated in a creative performance, choosing to assume risk, identifying business opportunities, gathering resources, initiating actions and maximizing opportunities. Stinhoff and Burgess [13] opined that entrepreneurship occurs when an individual develops a new venture, a new approach to an old business or idea, a unique way of giving the market place a product or service by using resources in a new way, under conditions of risk. Entrepreneurship education according to Lemechi and Anyakoha [10] refers to a carefully planned process leading to the acquisition of entrepreneurship skills for efficient and effective living. Entrepreneurship education is the key that opens the way to economic growth and development. It is the instrument that empowers youths to be in control of their future.

The aims and objectives of entrepreneurship education which centres on producing individuals who are not job seekers but job creators is in line with the attainment of the broad goal of secondary education as outlined in the National Policy on Education (NPE) 2004 which is to prepare the individual for useful living within the society. 
Chemistry as a branch of science has attained a unique position in the curriculum of schools as an essential part of general education for life [11]. Chemistry enables learners to understand what happens around them. Fahmy [4] states that the most interesting aspect of chemistry is that it apply to our daily lives. In order words chemistry is a real life science subject. The new chemistry curriculum for senior secondary schools was revised by the Federal Ministry of Education (FME) by 2009. The statement of intent of the revised edition of the senior secondary school chemistry curriculum [4] in Nigeria stated that "following Nigeria's endorsement for Education for All (EFA) and the millennium development goals (MDG) and subsequent adoption of a National Economic Empowerment and Development Strategies (NEEDs) it became imperative to update existing chemistry curriculum to cater for contemporary needs of the nation as a country aspiring to be amongst the first twenty economics in the world by the year 2020". The chemistry curriculum must be dynamic and move with the current developments all over the world. It therefore need to be reviewed from time to time to make room for changes in content form and also in the techniques in the pedagogy [3] . The aim of any reform in curriculum is to ensure that the programmes are always relevant to the nation's aspirations and meeting current technological development. The objectives of chemistry curriculum amongst other things includes to show chemistry and its link with industry, everyday life benefits and hazards; to provide a course which is complete for pupils not proceeding to higher education while its at the same time a reasonably adequate foundation for a post secondary chemistry course. The planners of chemistry curriculum recommend that the guided discovery approach be used in teaching. Apart from this guided discovery, there are other approaches which we can apply in teaching the contents in the curriculum to make for effective teaching and learning. Such approaches include use of field trip/ excursions, discussion, demonstration, Assignment/ Home work, laboratory work, conventional lectures, cooperative learning etc. An effective chemistry teacher should be selective in the use of different approaches in chemistry teaching depending on the subject matter and level of content development of the learner.

The main focus of this paper therefore is to identify those contents in the chemistry curriculum that can best promote the acquisition of entrepreneurial skills among senior secondary school students and also to find out the best approaches to be used in teaching those contents to promote the inculcation of these entrepreneurial skills in our students.

\section{Purpose of the Study}

The purpose of this study is to investigate the chemistry contents that can best promote the acquisition of entrepreneurial skills as well as the approaches for teaching them among our students.

\subsection{Research Questions}

The two research questions that guided the study are:

- Which of the topics in the senior secondary school chemistry curriculum can promote the inculcation of entrepreneurial skills in the students?

- What are the approaches that can be used to teach the topics in the senior secondary school chemistry curriculum for the promotion of entrepreneurship among students?

\subsection{Hypothesis}

There is no significant difference between the mean ratings of teachers and industrialists on the topics in the senior secondary chemistry curriculum that can promote the acquisition of entrepreneurial skills among the students.

\section{Method}

The design of this study is a survey: It is a survey because it is concerned with investigating events in their most natural settings and without manipulating of the variables. According to Ali [1] a survey is a study which seeks to document and describe what exists or the present status of existence or absence of what is being investigated. The study was carried out in Nsukka education zone of Enugu State, which is made up of three Local Governments areas namely Nsukka Local Government, Igbo-Etiti Local Government and Uzo-Uwani Local government Areas. The population of the study consisted of all the chemistry teachers in all the government owned secondary schools and all the industrialists in Nsukka education zone. Thirty secondary schools were randomly chosen out of the sixtyfive government owned secondary schools in the education zone. Thirty chemistry teachers and 10 industrialists were then randomly selected.

The instrument used for data collection is a self structured questionnaire. This is structured into two parts Part A sought information on the extent to which each of the contents in the chemistry curriculum promote the acquisition of entrepreneurial skills among the students. Part B sought information from the teachers only on the best approaches to be used in teaching the chemistry contents to make for the inculcation of entrepreneurial skills amongs our students. A four point rating scales of great extent, moderate extent, and little extent and not at all was provided for them to make their responses. The research instrument was subjected to scrutning by three science education experts from the Department of Science Education, University of Nigeria Nsukka. The researcher personally distributed the questionnaires to the chemistry teachers and the industrialists for their responses and also collected them from the respondents. The method ensured that all the 
questionnaires were returned. Mean and standard deviation were used in answering the research questions while the t-

\section{Results}

The results and statistical analysis of the data obtained are presented in the tables below:

Table 1. Means and Standard Deviation of the topics in the Chemistry curriculum that can promote the inculcation of entrepreneurial skills in the students

\begin{tabular}{|c|c|c|c|c|}
\hline $\mathbf{S} / \mathbf{N}$ & Chemistry & Mean & $\begin{array}{c}\text { Std. } \\
\text { Deviation }\end{array}$ & Decision \\
\hline 1. & $\begin{array}{l}\text { Concept of Matter: } \\
\text { Elements, Compounds } \\
\text { and Mixtures; } \\
\text { Differences between } \\
\text { compounds and mixtures }\end{array}$ & 1.07 & 0.27 & $\begin{array}{l}\text { Little } \\
\text { extent }\end{array}$ \\
\hline 2. & $\begin{array}{l}\text { Particulate nature of } \\
\text { Matter: Concept of atoms } \\
\text { and molecules Atomicity } \\
\text { of elements; Atomic } \\
\text { structure, sub-particles- } \\
\text { properties and functions } \\
\text { of the sub-particles }\end{array}$ & 1.256 & 0.43 & $\begin{array}{l}\text { Little } \\
\text { extent }\end{array}$ \\
\hline 3. & $\begin{array}{l}\text { Separation techniques for } \\
\text { mixtures: Filtration, } \\
\text { Evaporation Decantation } \\
\text { etc. }\end{array}$ & & & \\
\hline 4. & $\begin{array}{l}\text { Dalton's Atomic theory: } \\
\text { Its postulates; Atomic } \\
\text { number and atomic mass } \\
\text { number; Isotopy, Relative } \\
\text { atomic mass based on } \\
\text { carbon - } 12\end{array}$ & 1.37 & 0.49 & $\begin{array}{l}\text { Little } \\
\text { extent }\end{array}$ \\
\hline 5. & $\begin{array}{l}\text { Chemical symbols, } \\
\text { empirical and molecular } \\
\text { formula, chemical } \\
\text { equations }\end{array}$ & 1.70 & 0.65 & $\begin{array}{l}\text { Little } \\
\text { extent }\end{array}$ \\
\hline 6. & $\begin{array}{l}\text { Laws of chemical } \\
\text { combinations }\end{array}$ & 2.17 & 0.54 & $\begin{array}{l}\text { Little } \\
\text { extent }\end{array}$ \\
\hline 7. & The periodic table & 1.03 & 0.16 & $\begin{array}{l}\text { Little } \\
\text { extent }\end{array}$ \\
\hline 8. & Chemical bonding & 1.58 & 0.49 & $\begin{array}{l}\text { Little } \\
\text { extent }\end{array}$ \\
\hline 9. & $\begin{array}{l}\text { Kinetic theory of matter, } \\
\text { evidence in support of the } \\
\text { kinetic theory-diffusion, } \\
\text { osmosis etc }\end{array}$ & 2.17 & 0.55 & $\begin{array}{l}\text { Little } \\
\text { extent }\end{array}$ \\
\hline 10. & $\begin{array}{l}\text { The gas laws-boyle’s, } \\
\text { Charles etc }\end{array}$ & 1.75 & 0.63 & $\begin{array}{l}\text { Little } \\
\text { extent }\end{array}$ \\
\hline 11. & $\begin{array}{l}\text { Dalton's law of partial } \\
\text { pressure }\end{array}$ & 2.42 & 0.50 & $\begin{array}{l}\text { Little } \\
\text { extent }\end{array}$ \\
\hline 12. & $\begin{array}{l}\text { Graham's law of } \\
\text { diffusion }\end{array}$ & 2.55 & 0.50 & $\begin{array}{l}\text { Moderate } \\
\text { extent }\end{array}$ \\
\hline 13. & $\begin{array}{l}\text { The concept of mole, } \\
\text { Avogadro’s number and } \\
\text { molar volume of gases }\end{array}$ & 3.00 & 0.64 & $\begin{array}{l}\text { Great } \\
\text { extent }\end{array}$ \\
\hline 14. & Acids, bases and salts & 2.75 & 0.48 & $\begin{array}{l}\text { Moderate } \\
\text { extent }\end{array}$ \\
\hline
\end{tabular}

test statistics was employed in testing the sole hypothesis formulated for the study.

\begin{tabular}{|c|c|c|c|c|}
\hline 15. & $\begin{array}{l}\text { Water and solutions, } \\
\text { types of solutions, hard } \\
\text { water and methods of } \\
\text { softening water }\end{array}$ & 3.17 & 0.71 & $\begin{array}{l}\text { Great } \\
\text { extent }\end{array}$ \\
\hline 16. & $\begin{array}{l}\text { Saturated, unsaturated } \\
\text { and supersaturated } \\
\text { solutions; concept of } \\
\text { solubility and } \\
\text { calculations } \\
\end{array}$ & 3.42 & 0.43 & $\begin{array}{l}\text { Great } \\
\text { extent }\end{array}$ \\
\hline 17. & $\begin{array}{l}\text { Treatment of water for } \\
\text { public use; pollution of } \\
\text { water }\end{array}$ & 3.15 & 0.53 & $\begin{array}{l}\text { Great } \\
\text { extent }\end{array}$ \\
\hline 18. & $\begin{array}{l}\text { Carbon and its } \\
\text { compounds: Allotropes of } \\
\text { carbon }\end{array}$ & 2.57 & 0.50 & $\begin{array}{l}\text { Moderate } \\
\text { extent }\end{array}$ \\
\hline 19. & $\begin{array}{l}\text { Hydrocarbon and its main } \\
\text { classes }\end{array}$ & 2.47 & 0.55 & $\begin{array}{l}\text { Little } \\
\text { extent }\end{array}$ \\
\hline 20. & $\begin{array}{l}\text { Petroleum and its } \\
\text { fractions, octane number, } \\
\text { knocking of petrol, } \\
\text { reformation and cracking }\end{array}$ & 3.72 & 0.45 & $\begin{array}{l}\text { Great } \\
\text { extent }\end{array}$ \\
\hline 21. & $\begin{array}{l}\text { Industrial chemistry: } \\
\text { chemical industries, raw } \\
\text { materials and sources, } \\
\text { fine and heavy chemicals }\end{array}$ & 3.90 & 0.30 & $\begin{array}{l}\text { Great } \\
\text { extent }\end{array}$ \\
\hline 22. & $\begin{array}{l}\text { Periodic law, trends in } \\
\text { periodic properties }\end{array}$ & 1.10 & 0.30 & $\begin{array}{l}\text { Little } \\
\text { extent }\end{array}$ \\
\hline 23. & $\begin{array}{l}\text { Mass volume } \\
\text { relationships chemical } \\
\text { reactions and their uses in } \\
\text { determining the } \\
\text { stoichiometry of such } \\
\text { reactions }\end{array}$ & 3.00 & 0.64 & $\begin{array}{l}\text { Great } \\
\text { extent }\end{array}$ \\
\hline 24. & Electrolysis & 3.65 & 0.48 & $\begin{array}{l}\text { Great } \\
\text { extent }\end{array}$ \\
\hline 25. & $\begin{array}{l}\text { Quantitative electrolysis } \\
\text { of some compounds like } \\
\mathrm{NaCl}, \mathrm{HCl} \text {, etc. }\end{array}$ & 3.10 & 0.74 & $\begin{array}{l}\text { Great } \\
\text { extent }\end{array}$ \\
\hline 26. & Redox reactions & 2.32 & 0.48 & $\begin{array}{l}\text { Little } \\
\text { extent }\end{array}$ \\
\hline 27. & Electrochemical cells & 3.10 & 0.74 & $\begin{array}{l}\text { Great } \\
\text { extent }\end{array}$ \\
\hline 28. & $\begin{array}{l}\text { Rates of chemical } \\
\text { reactions }\end{array}$ & 2.92 & 0.53 & $\begin{array}{l}\text { Moderate } \\
\text { extent }\end{array}$ \\
\hline 29. & $\begin{array}{l}\text { Energy and reaction rates, } \\
\text { energy profile, } \\
\text { exothermic and } \\
\text { endothermic reactions }\end{array}$ & 3.07 & 0.66 & $\begin{array}{l}\text { Great } \\
\text { extent }\end{array}$ \\
\hline 30. & $\begin{array}{l}\text { Energy changes: Heat of } \\
\text { formation, combustion, } \\
\text { solution etc }\end{array}$ & 2.87 & 0.56 & $\begin{array}{l}\text { Moderate } \\
\text { extent }\end{array}$ \\
\hline 31. & $\begin{array}{l}\text { Source of energy for } \\
\text { chemical reactions, } \\
\text { enthalpy, entropy, free } \\
\text { energy }\end{array}$ & 2.10 & 0.54 & $\begin{array}{l}\text { Little } \\
\text { extent }\end{array}$ \\
\hline 32. & Chemical equilibrium & 2.49 & 0.49 & $\begin{array}{l}\text { Little } \\
\text { extent }\end{array}$ \\
\hline
\end{tabular}




\begin{tabular}{|c|c|c|c|c|}
\hline 33. & $\begin{array}{l}\text { Non metals and their } \\
\text { compounds: Hydrogen its } \\
\text { preparation, properties } \\
\text { and uses }\end{array}$ & 2.52 & 0.55 & $\begin{array}{l}\text { Moderate } \\
\text { extent }\end{array}$ \\
\hline 34. & $\begin{array}{l}\text { Oxygen: Preparation } \\
\text { properties and uses } \\
\text { oxides, air pollution- } \\
\text { causes, effects and } \\
\text { control }\end{array}$ & 3.00 & 0.45 & $\begin{array}{l}\text { Great } \\
\text { extent }\end{array}$ \\
\hline 35. & $\begin{array}{l}\text { Halogens: Position in the } \\
\text { periodic table, laboratory } \\
\text { preparation, properties } \\
\text { and reactions of chlorine }\end{array}$ & 2.90 & 0.52 & $\begin{array}{l}\text { Moderate } \\
\text { extent }\end{array}$ \\
\hline 36. & $\begin{array}{l}\text { Nitrogen: laboratory and } \\
\text { industrial preparation, } \\
\text { properties and uses of } \\
\text { nitrogen }\end{array}$ & 2.87 & 0.56 & $\begin{array}{l}\text { Moderate } \\
\text { extent }\end{array}$ \\
\hline 37. & $\begin{array}{l}\text { Sulphur: Allotropes and } \\
\text { uses, oxides of sulphur, } \\
\text { preparation, properties } \\
\text { and uses of } \mathrm{H}_{2} \mathrm{SO}_{4}\end{array}$ & 2.67 & 0.57 & $\begin{array}{l}\text { Moderate } \\
\text { extent }\end{array}$ \\
\hline 38. & $\begin{array}{l}\text { Organic chemistry: } \\
\text { Classification and } \\
\text { nomenclature of organic } \\
\text { compounds }\end{array}$ & 2.57 & 0.50 & $\begin{array}{l}\text { Great } \\
\text { extent }\end{array}$ \\
\hline 39. & $\begin{array}{l}\text { Homologous series: } \\
\text { Alkanes, alkanes, } \\
\text { alkynes, alkanols, } \\
\text { alkanmoic acids and } \\
\text { alkanoates, fats \& oils }\end{array}$ & 3.40 & 0.50 & $\begin{array}{l}\text { Great } \\
\text { extent }\end{array}$ \\
\hline 40. & $\begin{array}{l}\text { Soapless and soapy } \\
\text { detergents, amino acids, } \\
\text { aromatic hydrocarbons. }\end{array}$ & 3.40 & 0.50 & $\begin{array}{l}\text { Great } \\
\text { extent }\end{array}$ \\
\hline 41. & $\begin{array}{l}\text { Ethical, legal and social } \\
\text { issues }\end{array}$ & 3.47 & 0.51 & $\begin{array}{l}\text { Great } \\
\text { extent }\end{array}$ \\
\hline 42. & $\begin{array}{l}\text { Polymers and giant } \\
\text { molecules }\end{array}$ & 2.57 & 0.50 & $\begin{array}{l}\text { Moderate } \\
\text { extent }\end{array}$ \\
\hline 43 & $\begin{array}{l}\text { Carbohydrates: } \\
\text { classifications, properties } \\
\text { and uses }\end{array}$ & 3.27 & 0.68 & $\begin{array}{l}\text { Great } \\
\text { extent }\end{array}$ \\
\hline 44 & $\begin{array}{l}\text { Electronic structure of the } \\
\text { atom: Qualitative } \\
\text { treatment of the wave } \\
\text { nature of light, orbitals, } \\
\text { origin and shapes of S, P, } \\
\text { d and f orbitals. }\end{array}$ & 2.10 & 0.67 & $\begin{array}{l}\text { Little } \\
\text { extent }\end{array}$ \\
\hline 45. & $\begin{array}{l}\text { Quantum numbers, rules } \\
\text { and principles of filling } \\
\text { electrons. }\end{array}$ & 1.82 & 0.64 & $\begin{array}{l}\text { Little } \\
\text { extent }\end{array}$ \\
\hline 46. & Radioactivity & 2.55 & 0.50 & $\begin{array}{l}\text { Moderate } \\
\text { extent }\end{array}$ \\
\hline 47. & $\begin{array}{l}\text { Metals and their } \\
\text { compounds }\end{array}$ & 3.81 & 0.29 & $\begin{array}{l}\text { Great } \\
\text { extent }\end{array}$ \\
\hline 48. & $\begin{array}{l}\text { Qualitative analysis: } \\
\text { Identification of cations } \\
\text { and anions }\end{array}$ & 3.85 & 0.36 & $\begin{array}{l}\text { Great } \\
\text { extent }\end{array}$ \\
\hline 49. & $\begin{array}{l}\text { Quantitative analysis: } \\
\text { Acid-base titrations. }\end{array}$ & 3.81 & 0.29 & $\begin{array}{l}\text { Great } \\
\text { extent }\end{array}$ \\
\hline 50. & $\begin{array}{l}\text { Preparation of standard } \\
\text { solutions }\end{array}$ & 3.92 & 0.19 & $\begin{array}{l}\text { Great } \\
\text { extent }\end{array}$ \\
\hline
\end{tabular}

Table 1 indicates that some topics in the chemistry curriculum can promote the inculcation of entrepreneurial skills in students while some others do not. Using 2.5 at cut off points, thirty three major topics namely separation techniques, Graham's law, water and solutions, treatment of water for public use, mass volume relationships in chemical reactions, Energy and energy changes, Acids Bases and salt, rates of reactions and equilibrium systems, chemistry of carbon compounds, industrial chemistry etc promote the inculcation of entrepreneurial skills in students while seventeen topics namely. Concept of matter, particulate nature of matter, periodic table and chemical bonding etc do not promote the inculcation of entrepreneurial skills in students.

Table 2. Means and Standard Deviation of the approaches that can be used in teaching the contents in the senior secondary chemistry curriculum to promote

the inculcation of entrepreneurial skills among the students

\begin{tabular}{|l|l|l|l|}
\hline S/N & \multicolumn{1}{|c|}{ Teaching Approaches } & Means & $\begin{array}{l}\text { Standard } \\
\text { Deviation }\end{array}$ \\
\hline 1. & $\begin{array}{l}\text { Use of Home Work/ } \\
\text { Assignments }\end{array}$ & 2.80 & 0.53 \\
\hline 2. & Field trip/Excursion & 3.60 & 0.50 \\
\hline 3. & Discussion & 2.30 & 0.55 \\
\hline 4. & Use of practical activities & 3.40 & 0.50 \\
\hline 5. & Inquiry approach & 3.33 & 0.55 \\
\hline 6. & Demonstration & 2.50 & 0.51 \\
\hline 7. & Concept mapping & 2.83 & 0.53 \\
\hline 8. & Cooperative learning & 3.00 & 0.59 \\
\hline 9. & Conventional lecture & 1.57 & 0.50 \\
\hline 10. & Guided discovery & 3.70 & 0.47 \\
\hline
\end{tabular}

Table 2 revealed that the use of field trip/excursion, use of practical activities, inquiry approach, cooperative learning, use of home work/assignment and guided discovery approach promote the inculcation of entrepreneurial skills in our students to a great extent. While the use of demonstration and concept mapping promote the inculcation of entrepreneurship to a moderate extent. The use of discussion and conventional lecture do not promote the inculcation of entrepreneurship among our students.

Table 3. t-test Difference in mean scores in teachers and industrialists with regards to contents in chemistry curriculum that promote the acquisition of entrepreneurial skills among senior secondary school students

\begin{tabular}{|c|c|c|c|c|c|c|c|c|}
\hline $\begin{array}{c}\text { Ite } \\
\text { m }\end{array}$ & Status & $\mathbf{N}$ & $\begin{array}{c}\text { Mea } \\
\text { n }\end{array}$ & $\begin{array}{c}\text { Stan } \\
\text { d. } \\
\text { Dev. }\end{array}$ & t & df & Sig & $\begin{array}{c}\text { Decisi } \\
\text { on }\end{array}$ \\
\hline 1. & $\begin{array}{l}\text { Chemistry teacher } \\
\text { Industrial Worker }\end{array}$ & $\begin{array}{l}30 \\
10\end{array}$ & $\begin{array}{l}1.07 \\
1.10\end{array}$ & $\begin{array}{l}0.25 \\
0.32\end{array}$ & -0.34 & 38 & 0.74 & $\begin{array}{l}\text { Not } \\
\text { signifi } \\
\text { cant }\end{array}$ \\
\hline 2. & $\begin{array}{l}\text { Chemistry teacher } \\
\text { Industrial Worker }\end{array}$ & $\begin{array}{l}30 \\
10\end{array}$ & $\begin{array}{l}1.23 \\
1.30\end{array}$ & $\begin{array}{l}0.43 \\
0.48\end{array}$ & -041 & 38 & 0.68 & $\begin{array}{l}\text { Not } \\
\text { Signifi } \\
\text { cant }\end{array}$ \\
\hline 3. & Chemistry teacher & 30 & 3.43 & 0.50 & 0.73 & 38 & 0.69 & Not \\
\hline
\end{tabular}




\begin{tabular}{|c|c|c|c|c|c|c|c|c|}
\hline & Industrial Worker & 10 & 3.30 & 0.48 & & & & $\begin{array}{l}\text { Signifi } \\
\text { cant }\end{array}$ \\
\hline 4. & $\begin{array}{l}\text { Chemistry teacher } \\
\text { Industrial Worker }\end{array}$ & $\begin{array}{l}30 \\
10\end{array}$ & $\begin{array}{l}1.40 \\
1.27\end{array}$ & $\begin{array}{l}0.38 \\
0.32\end{array}$ & 0.55 & 38 & 0.58 & $\begin{array}{l}\text { Not } \\
\text { Signifi } \\
\text { cant }\end{array}$ \\
\hline 5. & $\begin{array}{l}\text { Chemistry teacher } \\
\text { Industrial Worker }\end{array}$ & $\begin{array}{l}30 \\
10\end{array}$ & $\begin{array}{l}2.53 \\
2.50\end{array}$ & $\begin{array}{l}0.57 \\
0.52\end{array}$ & 0.16 & 38 & 0.87 & $\begin{array}{l}\text { Not } \\
\text { Signifi } \\
\text { cant }\end{array}$ \\
\hline 6. & $\begin{array}{l}\text { Chemistry teacher } \\
\text { Industrial Worker }\end{array}$ & $\begin{array}{l}30 \\
10\end{array}$ & $\begin{array}{l}2.30 \\
2.80\end{array}$ & $\begin{array}{l}0.53 \\
0.42\end{array}$ & 2.68 & 38 & 0.01 & $\begin{array}{l}\text { Signifi } \\
\text { cant }\end{array}$ \\
\hline 7. & $\begin{array}{l}\text { Chemistry teacher } \\
\text { Industrial Worker }\end{array}$ & $\begin{array}{l}30 \\
10\end{array}$ & $\begin{array}{l}1.00 \\
1.10\end{array}$ & $\begin{array}{l}0.00 \\
0.31\end{array}$ & -1.78 & 38 & 0.08 & $\begin{array}{l}\text { Not } \\
\text { Signifi } \\
\text { cant }\end{array}$ \\
\hline 8. & $\begin{array}{l}\text { Chemistry teacher } \\
\text { Industrial Worker }\end{array}$ & $\begin{array}{l}30 \\
10\end{array}$ & $\begin{array}{l}1.58 \\
1.54\end{array}$ & $\begin{array}{l}0.50 \\
0.49\end{array}$ & 0.29 & 38 & 0.77 & $\begin{array}{l}\text { Not } \\
\text { Signifi } \\
\text { cant }\end{array}$ \\
\hline 9. & $\begin{array}{l}\text { Chemistry teacher } \\
\text { Industrial Worker }\end{array}$ & $\begin{array}{l}30 \\
10\end{array}$ & $\begin{array}{l}2.30 \\
1.80\end{array}$ & $\begin{array}{l}0.53 \\
0.42\end{array}$ & 2.68 & 38 & 0.59 & $\begin{array}{l}\text { Not } \\
\text { Signifi } \\
\text { cant }\end{array}$ \\
\hline 10. & $\begin{array}{l}\text { Chemistry teacher } \\
\text { Industrial Worker }\end{array}$ & $\begin{array}{l}30 \\
10\end{array}$ & $\begin{array}{l}1.80 \\
1.60\end{array}$ & $\begin{array}{l}0.66 \\
0.52\end{array}$ & 0.87 & 38 & 0.71 & $\begin{array}{l}\text { Not } \\
\text { Signifi } \\
\text { cant }\end{array}$ \\
\hline 11. & $\begin{array}{l}\text { Chemistry teacher } \\
\text { Industrial Worker }\end{array}$ & $\begin{array}{l}30 \\
10\end{array}$ & $\begin{array}{l}2.40 \\
2.60\end{array}$ & $\begin{array}{l}0.55 \\
0.50\end{array}$ & -0.80 & 38 & 0.40 & $\begin{array}{l}\text { Not } \\
\text { Signifi } \\
\text { cant }\end{array}$ \\
\hline 12. & $\begin{array}{l}\text { Chemistry teacher } \\
\text { Industrial Worker }\end{array}$ & $\begin{array}{l}30 \\
10\end{array}$ & $\begin{array}{l}2.53 \\
2.60\end{array}$ & $\begin{array}{l}0.51 \\
0.52\end{array}$ & -0.36 & 38 & 0.72 & $\begin{array}{l}\text { Not } \\
\text { Signifi } \\
\text { cant }\end{array}$ \\
\hline 13. & $\begin{array}{l}\text { Chemistry teacher } \\
\text { Industrial Worker }\end{array}$ & $\begin{array}{l}30 \\
10\end{array}$ & $\begin{array}{l}3.07 \\
2.80\end{array}$ & $\begin{array}{l}0.64 \\
0.63\end{array}$ & 1.15 & 38 & 0.26 & $\begin{array}{l}\text { Not } \\
\text { Signifi } \\
\text { cant }\end{array}$ \\
\hline 14. & $\begin{array}{l}\text { Chemistry teacher } \\
\text { Industrial Worker }\end{array}$ & $\begin{array}{l}30 \\
10\end{array}$ & $\begin{array}{l}2.80 \\
2.60\end{array}$ & $\begin{array}{l}0.47 \\
0.48\end{array}$ & $\begin{array}{l}1.12 \\
1.12\end{array}$ & 38 & 0.27 & $\begin{array}{l}\text { Not } \\
\text { Signifi } \\
\text { cant }\end{array}$ \\
\hline 15. & $\begin{array}{l}\text { Chemistry teacher } \\
\text { Industrial Worker }\end{array}$ & $\begin{array}{l}30 \\
10\end{array}$ & $\begin{array}{l}3.20 \\
3.10\end{array}$ & $\begin{array}{l}0.71 \\
0.74\end{array}$ & 0.38 & 38 & 0.70 & $\begin{array}{l}\text { Not } \\
\text { Signifi } \\
\text { cant }\end{array}$ \\
\hline 16. & $\begin{array}{l}\text { Chemistry teacher } \\
\text { Industrial Worker }\end{array}$ & $\begin{array}{l}30 \\
10\end{array}$ & $\begin{array}{l}3.47 \\
3.30\end{array}$ & $\begin{array}{l}0.43 \\
0.42\end{array}$ & 1.07 & 38 & 0.30 & $\begin{array}{l}\text { Not } \\
\text { Signifi } \\
\text { cant }\end{array}$ \\
\hline 17. & $\begin{array}{l}\text { Chemistry teacher } \\
\text { Industrial Worker }\end{array}$ & $\begin{array}{l}30 \\
10\end{array}$ & $\begin{array}{l}3.20 \\
3.00\end{array}$ & $\begin{array}{l}0.55 \\
0.47\end{array}$ & -1.03 & 38 & 0.31 & $\begin{array}{l}\text { Not } \\
\text { Signifi } \\
\text { cant }\end{array}$ \\
\hline 18. & $\begin{array}{l}\text { Chemistry teacher } \\
\text { Industrial Worker }\end{array}$ & $\begin{array}{l}30 \\
10\end{array}$ & $\begin{array}{l}2.57 \\
2.60\end{array}$ & $\begin{array}{l}0.50 \\
0.52\end{array}$ & -1.80 & 38 & 0.86 & $\begin{array}{l}\text { Not } \\
\text { Signifi } \\
\text { cant }\end{array}$ \\
\hline 19. & $\begin{array}{l}\text { Chemistry teacher } \\
\text { Industrial Worker }\end{array}$ & $\begin{array}{l}30 \\
10\end{array}$ & $\begin{array}{l}2.51 \\
2.44\end{array}$ & $\begin{array}{l}0.49 \\
0.46\end{array}$ & 0.37 & 38 & 0.71 & $\begin{array}{l}\text { Not } \\
\text { Signifi } \\
\text { cant }\end{array}$ \\
\hline 20. & $\begin{array}{l}\text { Chemistry teacher } \\
\text { Industrial Worker } \\
\end{array}$ & $\begin{array}{l}30 \\
10 \\
\end{array}$ & $\begin{array}{l}3.63 \\
4.00 \\
\end{array}$ & $\begin{array}{l}0.49 \\
0.00 \\
\end{array}$ & -2.34 & 38 & 0.02 & $\begin{array}{l}\text { Signifi } \\
\text { cant }\end{array}$ \\
\hline 21. & $\begin{array}{l}\text { Chemistry teacher } \\
\text { Industrial Worker }\end{array}$ & $\begin{array}{l}30 \\
10\end{array}$ & $\begin{array}{l}3.90 \\
3.91\end{array}$ & $\begin{array}{l}0.30 \\
0.31\end{array}$ & 0.00 & 38 & 1.00 & $\begin{array}{l}\text { Not } \\
\text { Signifi } \\
\text { cant }\end{array}$ \\
\hline 22. & $\begin{array}{l}\text { Chemistry teacher } \\
\text { Industrial Worker }\end{array}$ & $\begin{array}{l}30 \\
10\end{array}$ & $\begin{array}{l}1.13 \\
1.00\end{array}$ & $\begin{array}{l}0.35 \\
0.00\end{array}$ & 1.21 & 38 & 0.23 & $\begin{array}{l}\text { Not } \\
\text { Signifi } \\
\text { cant }\end{array}$ \\
\hline 23. & $\begin{array}{l}\text { Chemistry teacher } \\
\text { Industrial Worker }\end{array}$ & $\begin{array}{l}30 \\
10\end{array}$ & $\begin{array}{l}3.07 \\
2.87\end{array}$ & $\begin{array}{l}0.64 \\
0.63\end{array}$ & 1.15 & 38 & 0.26 & $\begin{array}{l}\text { Not } \\
\text { Signifi } \\
\text { cant }\end{array}$ \\
\hline 24. & $\begin{array}{l}\text { Chemistry teacher } \\
\text { Industrial Worker }\end{array}$ & $\begin{array}{l}30 \\
10\end{array}$ & $\begin{array}{l}3.70 \\
3.50\end{array}$ & $\begin{array}{l}0.47 \\
0.53\end{array}$ & 1.14 & 38 & 0.26 & $\begin{array}{l}\text { Not } \\
\text { Signifi } \\
\text { cant }\end{array}$ \\
\hline 25. & $\begin{array}{l}\text { Chemistry teacher } \\
\text { Industrial Worker }\end{array}$ & $\begin{array}{l}30 \\
10\end{array}$ & $\begin{array}{l}2.93 \\
3.20\end{array}$ & $\begin{array}{l}0.46 \\
0.42\end{array}$ & -1.64 & 38 & 0.26 & $\begin{array}{l}\text { Not } \\
\text { Signifi } \\
\text { cant }\end{array}$ \\
\hline 26. & $\begin{array}{l}\text { Chemistry teacher } \\
\text { Industrial Worker }\end{array}$ & $\begin{array}{l}30 \\
10 \\
\end{array}$ & $\begin{array}{l}2.70 \\
3.40 \\
\end{array}$ & $\begin{array}{l}0.49 \\
0.52 \\
\end{array}$ & -4.00 & 38 & 0.00 & $\begin{array}{l}\text { Signifi } \\
\text { cant }\end{array}$ \\
\hline 27. & $\begin{array}{l}\text { Chemistry teacher } \\
\text { Industrial Worker }\end{array}$ & $\begin{array}{l}30 \\
10 \\
\end{array}$ & $\begin{array}{l}3.27 \\
2.60 \\
\end{array}$ & $\begin{array}{l}0.74 \\
0.52 \\
\end{array}$ & 2.63 & 38 & 0.01 & $\begin{array}{l}\text { Signifi } \\
\text { cant }\end{array}$ \\
\hline 28. & $\begin{array}{l}\text { Chemistry teacher } \\
\text { Industrial Worker }\end{array}$ & $\begin{array}{l}30 \\
10\end{array}$ & $\begin{array}{l}2.83 \\
3.20\end{array}$ & $\begin{array}{l}0.53 \\
0.42\end{array}$ & -1.98 & 38 & 0.06 & $\begin{array}{l}\text { Not } \\
\text { Signifi } \\
\text { cant }\end{array}$ \\
\hline 29. & $\begin{array}{l}\text { Chemistry teacher } \\
\text { Industrial Worker }\end{array}$ & $\begin{array}{l}30 \\
10\end{array}$ & $\begin{array}{l}3.03 \\
3.20\end{array}$ & $\begin{array}{l}0.72 \\
0.42\end{array}$ & -6.91 & 38 & 0.49 & $\begin{array}{l}\text { Not } \\
\text { Signifi } \\
\text { cant }\end{array}$ \\
\hline 30. & $\begin{array}{l}\text { Chemistry teacher } \\
\text { Industrial Worker }\end{array}$ & $\begin{array}{l}30 \\
10 \\
\end{array}$ & $\begin{array}{l}3.20 \\
2.40\end{array}$ & $\begin{array}{l}0.66 \\
0.52 \\
\end{array}$ & 3.46 & 38 & 0.01 & $\begin{array}{l}\text { Signifi } \\
\text { cant }\end{array}$ \\
\hline
\end{tabular}

\begin{tabular}{|c|c|c|c|c|c|c|c|c|}
\hline 31. & $\begin{array}{l}\text { Chemistry teacher } \\
\text { Industrial Worker }\end{array}$ & $\begin{array}{l}30 \\
10\end{array}$ & $\begin{array}{l}2.10 \\
2.10\end{array}$ & $\begin{array}{l}0.55 \\
0.57\end{array}$ & 0.00 & 38 & 1.00 & $\begin{array}{l}\text { Not } \\
\text { Signifi } \\
\text { cant }\end{array}$ \\
\hline 32. & $\begin{array}{l}\text { Chemistry teacher } \\
\text { Industrial Worker }\end{array}$ & $\begin{array}{l}30 \\
10\end{array}$ & $\begin{array}{l}2.87 \\
2.90\end{array}$ & $\begin{array}{l}0.50 \\
0.50\end{array}$ & -0.16 & 38 & 0.87 & $\begin{array}{l}\text { Not } \\
\text { Signifi } \\
\text { cant }\end{array}$ \\
\hline 33. & $\begin{array}{l}\text { Chemistry teacher } \\
\text { Industrial Worker }\end{array}$ & $\begin{array}{l}30 \\
10\end{array}$ & $\begin{array}{l}2.50 \\
2.60\end{array}$ & $\begin{array}{l}0.51 \\
0.52\end{array}$ & -0.51 & 38 & 0.60 & $\begin{array}{l}\text { Not } \\
\text { Signifi } \\
\text { cant }\end{array}$ \\
\hline 34. & $\begin{array}{l}\text { Chemistry teacher } \\
\text { Industrial Worker }\end{array}$ & $\begin{array}{l}30 \\
10\end{array}$ & $\begin{array}{l}3.40 \\
3.06\end{array}$ & $\begin{array}{l}0.56 \\
0.52\end{array}$ & -0.99 & 38 & 0.32 & $\begin{array}{l}\text { Not } \\
\text { Signifi } \\
\text { cant }\end{array}$ \\
\hline 35. & $\begin{array}{l}\text { Chemistry teacher } \\
\text { Industrial Worker }\end{array}$ & $\begin{array}{l}30 \\
10\end{array}$ & $\begin{array}{l}2.85 \\
3.05\end{array}$ & $\begin{array}{l}0.54 \\
0.44\end{array}$ & -1.05 & 38 & 0.30 & $\begin{array}{l}\text { Not } \\
\text { Signifi } \\
\text { cant }\end{array}$ \\
\hline 36. & $\begin{array}{l}\text { Chemistry teacher } \\
\text { Industrial Worker }\end{array}$ & $\begin{array}{l}30 \\
10\end{array}$ & $\begin{array}{l}2.87 \\
2.90\end{array}$ & $\begin{array}{l}0.57 \\
0.57\end{array}$ & -1.60 & 38 & 0.87 & $\begin{array}{l}\text { Not } \\
\text { Signifi } \\
\text { cant }\end{array}$ \\
\hline 37. & $\begin{array}{l}\text { Chemistry teacher } \\
\text { Industrial Worker }\end{array}$ & $\begin{array}{l}30 \\
10\end{array}$ & $\begin{array}{l}2.63 \\
2.80\end{array}$ & $\begin{array}{l}0.56 \\
0.63\end{array}$ & -7.94 & 38 & 0.43 & $\begin{array}{l}\text { Not } \\
\text { Signifi } \\
\text { cant }\end{array}$ \\
\hline 38. & $\begin{array}{l}\text { Chemistry teacher } \\
\text { Industrial Worker }\end{array}$ & $\begin{array}{l}30 \\
10\end{array}$ & $\begin{array}{l}2.50 \\
2.60\end{array}$ & $\begin{array}{l}0.50 \\
0.52\end{array}$ & -0.18 & 38 & 0.86 & $\begin{array}{l}\text { Not } \\
\text { Signifi } \\
\text { cant }\end{array}$ \\
\hline 39. & $\begin{array}{l}\text { Chemistry teacher } \\
\text { Industrial Worker }\end{array}$ & $\begin{array}{l}30 \\
10\end{array}$ & $\begin{array}{l}3.27 \\
3.20\end{array}$ & $\begin{array}{l}0.45 \\
0.42\end{array}$ & 0.41 & 38 & 0.68 & $\begin{array}{l}\text { Not } \\
\text { Signifi } \\
\text { cant }\end{array}$ \\
\hline 40. & $\begin{array}{l}\text { Chemistry teacher } \\
\text { Industrial Worker }\end{array}$ & $\begin{array}{l}30 \\
10\end{array}$ & $\begin{array}{l}3.37 \\
3.00\end{array}$ & $\begin{array}{l}0.49 \\
1.05\end{array}$ & 1.50 & 38 & 0.14 & $\begin{array}{l}\text { Not } \\
\text { Signifi } \\
\text { cant }\end{array}$ \\
\hline 41. & $\begin{array}{l}\text { Chemistry teacher } \\
\text { Industrial Worker }\end{array}$ & $\begin{array}{l}30 \\
10\end{array}$ & $\begin{array}{l}3.53 \\
3.30\end{array}$ & $\begin{array}{l}0.50 \\
0.48\end{array}$ & 1.27 & 38 & 0.21 & $\begin{array}{l}\text { Not } \\
\text { Signifi } \\
\text { cant }\end{array}$ \\
\hline 42. & $\begin{array}{l}\text { Chemistry teacher } \\
\text { Industrial Worker }\end{array}$ & $\begin{array}{l}30 \\
10\end{array}$ & $\begin{array}{l}1.27 \\
1.10\end{array}$ & $\begin{array}{l}0.45 \\
0.31\end{array}$ & 1.08 & 38 & 0.22 & $\begin{array}{l}\text { Not } \\
\text { Signifi } \\
\text { cant }\end{array}$ \\
\hline 43. & $\begin{array}{l}\text { Chemistry teacher } \\
\text { Industrial Worker }\end{array}$ & $\begin{array}{l}30 \\
10\end{array}$ & $\begin{array}{l}1.20 \\
1.40\end{array}$ & $\begin{array}{l}0.41 \\
0.51\end{array}$ & -1.26 & 38 & 0.29 & $\begin{array}{l}\text { Not } \\
\text { Signifi } \\
\text { cant }\end{array}$ \\
\hline 44. & $\begin{array}{l}\text { Chemistry teacher } \\
\text { Industrial Worker }\end{array}$ & $\begin{array}{l}30 \\
10\end{array}$ & $\begin{array}{l}1.20 \\
1.40\end{array}$ & $\begin{array}{l}0.41 \\
0.51\end{array}$ & -1.26 & 38 & 0.29 & $\begin{array}{l}\text { Not } \\
\text { Signifi } \\
\text { cant }\end{array}$ \\
\hline 45. & $\begin{array}{l}\text { Chemistry teacher } \\
\text { Industrial Worker }\end{array}$ & $\begin{array}{l}30 \\
10\end{array}$ & $\begin{array}{l}1.27 \\
1.20\end{array}$ & $\begin{array}{l}0.45 \\
0.31\end{array}$ & 1.08 & 38 & 0.22 & $\begin{array}{l}\text { Not } \\
\text { Signifi } \\
\text { cant }\end{array}$ \\
\hline 46. & $\begin{array}{l}\text { Chemistry teacher } \\
\text { Industrial Worker }\end{array}$ & $\begin{array}{l}30 \\
10\end{array}$ & $\begin{array}{l}3.87 \\
3.80\end{array}$ & $\begin{array}{l}0.35 \\
0.32\end{array}$ & 0.50 & 38 & 0.62 & $\begin{array}{l}\text { Not } \\
\text { Signifi } \\
\text { cant }\end{array}$ \\
\hline 47. & $\begin{array}{l}\text { Chemistry teacher } \\
\text { Industrial Worker }\end{array}$ & $\begin{array}{l}30 \\
10\end{array}$ & $\begin{array}{l}4.00 \\
4.00\end{array}$ & $\begin{array}{l}0.00 \\
0.00\end{array}$ & -1.03 & 38 & 0.31 & $\begin{array}{l}\text { Not } \\
\text { Signifi } \\
\text { cant }\end{array}$ \\
\hline 48 & $\begin{array}{l}\text { Chemistry teacher } \\
\text { Industrial Worker }\end{array}$ & $\begin{array}{l}30 \\
10\end{array}$ & $\begin{array}{l}3.90 \\
4.00\end{array}$ & $\begin{array}{l}0.31 \\
0.00\end{array}$ & -1.79 & 38 & 0.00 & $\begin{array}{l}\text { Not } \\
\text { Signifi } \\
\text { cant }\end{array}$ \\
\hline
\end{tabular}

The result on table 3 shows that no significant difference was recorded between the perceptions of teachers and industrial workers towards the contents in the senior secondary chemistry curriculum that can promote entrepreneurial skills among the students. The null hypothesis is therefore accepted.

\section{Discussion of Findings}

The results of the findings indicates that thirty three out of the fifty major topics in the senior secondary school chemistry curriculum namely separation techniques, Graham's law, water and solutions, treatment of water for public use, mass volume relationships, energy and energy changes, acids bases and salts, solubility of substances, Rates of reactions and equilibrium systems, chemistry of carbon compounds, 
industrial chemistry etc promote the inculcation of entrepreneurial skills among our students while seventeen of the contents namely concept of matter, particulate nature of matter, periodic table, chemical bonding, redox reactions etc do not. The finding of this result is an affirmation of the objectives of senior secondary chemistry curriculum which among others is to show chemistry and its link with industry, everyday life benefits and hazards, to provide a course which is complete for pupils not proceeding to higher education while its at the same time a reasonably adequate foundation for a post secondary chemistry course. [3]. Thus, proper studying of the subject chemistry will actually prepare an individual to be able to create a job for himself/herself after school, especially now that unemployment is the order of the day. This notwithstanding, there is the need to include more topics from entrepreneurial education proper which is solely tailored towards training the students on how to acquire the skills of entrepreneurship.

The findings on the approaches to be used in teaching the contents in the senior secondary chemistry curriculum revealed that the use of field trip/excursion, use of practical activities, inquiry approach, cooperative learning, use of home work/assignment and guided discovery approach promote the inculcation of entrepreneurial skills in our students to a great extent. While the use of demonstration and concept mapping promote the inculcation of entrepreneurship to a moderate extent. The use of discussion and conventional lecture do not promote the inculcation of entrepreneurship among our students. The finding agree with the result of the research by Bonwell and Elson [2] which showed that active learning strategies are comparable to lectures for achieving content mastery, but superior to lectures for developing critical thinking.

The finding is also in agreement with the assertion of Osisioma [9] who maintains that students learn meaningfully when they are actively involved in the learning process. This is moreso when they work in small groups and have the opportunity to negotiate meaning and construct conceptual understanding in a community of learners thereby making education more relevant and self reliant which are the prerequisites to entrepreneurial skills.

\section{Conclusion and Recommendations}

From the results of the research, it can be seen that many of the contents in the senior secondary chemistry curriculum are designed to facilitate the acquisition of entrepreneurial skills among the students. The teachers should therefore try as much as possible to combine the appropriate teaching approaches as identified by the researcher to make for the inculcation of these skills among the students.

Based on these findings, the following recommendations were made:

- Serving chemistry teachers should be sponsored on a regular basis to workshops, seminars and conferences for continuous training in the skills for using the current teaching approaches that promote creative and manipulative skills among the students and hence prepares them for a self reliant life in the wider society.

- The curriculum designed for senior secondary chemistry education should be restructured in such a way that apart from the already existing contents, it should also include courses in entrepreneurship education proper.

- Well-equipped laboratories must be provided in schools. These laboratories should have enough equipment, materials and even improvised materials. Laboratory technologists, technicians and laboratory assistances should be provided and re-trained in improvisation.

\section{References}

[1] Ali, A. (1992). Methods and materials for science teaching, Journal of Science Teacher Association of Nigeria 34(1 \& 2).

[2] Bonwell, C.C. \& Elson J.A. (2000). Active learning creating Excitement in the classroom. Journal of Science Teacher's Association of Nigeria

[3]Eya, N.M (2011) Chemistry Curriculum Reforms in the $21^{\text {st }}$ Century. The Need for Human Resource Development. 52 Annual Conference of Science Teachers Association of Nigeria ( STAN) proceedings. PP 255-260

[4] Fahmy, A. F. M. (2000), Systemic Approach to teaching and learning chemistry for $21^{\text {st }}$ century rkshop on New Trend in chemistry. Buda pest, Hungary, August 5-10, 2000. fahmy@online.com.eg.

[5] Federal Ministry of Education (FME) (2009). National; Curriculum for Senior Secondary Schools. NERDC Lagos.

[6] Federal Republic of Nigeria (2004). National Policy on Education NERDC press, Nigeria: Yabba-Lagos

[7] Igbo,C.A (2004).Entrepreneur Initiatives in colleges of Education Programme.Paper presented at the workshop on capacity building for lecturers in colleges of education $19^{\text {th }}-25^{\text {th }}$ sept 2004

[8] Igbo, C.A.(2006). Developing Entrepreneurship through Entrepreneurship Education In Elizabeth , U.A ( Ed) Entrepreneurship Education and wealth creation strategies.Great AT Express Publishers Nsukka

[9] Ikeme and Onuh (2006): Practical tips to successful entrepreneurship. Entrepreneurship Education and Wealth creation strategies: Practical tips for economic empowerment and survival. Great AT Express Publishers Nsukka.

[10] Lemechi, S.N. \& Anyakoha, E.U. (2002): Teaching Entrepreneurship in Vocational Education: An Imperative Towards Sustainable Educational Development in Nigeria: Nigerian Journal of curriculum studies 9(1). 
[11] Okafor, P.N. (1998). Scientific literacy: A vehicle for national development. A Journal of the School of Technical Education, Federal College of Education (Technical). AKOKA (PP74-88) 2(1) Akoka Books.

[12] Osisioma, I. (2005). Active learning strategies for the science classrooms (workshop) proceedings of the $46^{\text {th }}$ Annual conference 2005. Uchenna Nzewi. I. Heiemann Educational Books (Nigeria) Plc.

[13] Steinhoff, D \& Burgess, J.F. (1993). Small Business Management Fundamentals, (6 ${ }^{\text {th }}$ ed). New York: McGraw Hill International. 